\title{
Validation of a frailty index in older cancer patients with solid tumours
}

\author{
A. L. McCarthy ${ }^{1,3^{*}}$ (D, N. M. Peel ${ }^{4}$, K. M. Gillespie ${ }^{2}$, R. Berry ${ }^{3}$, E. Walpole ${ }^{3}$, P. Yates ${ }^{2}$ and R. E. Hubbard ${ }^{4}$
}

\begin{abstract}
Background: Frailty is an indicator of physiological reserve in older people. In non-cancer settings, frailty indices are reliable predictors of adverse health outcomes. The aims of this study were to 1) derive and validate a frailty index (FI) from comprehensive geriatric assessment (CGA) data obtained in the solid tumour chemotherapy setting, and 2) to explore whether the FI-CGA could predict chemotherapy decisions and survival in older cancer patients with solid tumours.

Methods: Prospective cohort study of a consecutive series sample of 175 cancer patients aged 65 and older with solid tumours. A frailty index was calculated using an accumulated deficits model, coding items from the comprehensive geriatric assessment tool administered prior to chemotherapy decision-making. The domains of physical and cognitive functioning, nutrition, mood, basic and instrumental activities of daily living, and comorbidities were incorporated as deficits into the model.

Results: The FI-CGA had a right-skewed distribution, with median (interquartile range) of 0.27 (0.21-0.39). The $99 \%$ limit to deficit accumulation was below the theoretical maximum of 1.0, at 0.75 . The FI-CGA was significantly related $(p<0.001)$ to vulnerability as assessed by the Vulnerable Elders Survey-13 and to medical oncologists' assessments of fitness or vulnerability to treatment. Baseline frailty as determined by the FI-CGA was also associated with treatment decisions (Treatment Terminated, Treatment Completed, No Planned Treatment) $(p<0.001)$, with the No Planned Treatment group significantly frailer than the other two groups.
\end{abstract}

Conclusion: The FI-CGA is a potentially useful adjunct to cancer clinical decision-making that could predict chemotherapy outcomes in older patients with solid tumours.

Keywords: Geriatric oncology, Frailty, Comprehensive geriatric assessment, Chemotherapy

\section{Background}

Research interest in recent years has focused on methods to assess older cancer patients' fitness for chemotherapy and to predict their potential responses to it [1-3]. Accurate prediction of chemotherapy outcomes could obviate current concerns about over- and under-treatment in this cohort [4-6]. An approach that has received consistent attention is comprehensive geriatric assessment (CGA). In this paper we report the results of a prospective cohort study. The aim of the study was to derive and validate a frailty index (FI) from

\footnotetext{
* Correspondence: alexandra.mccarthy@auckland.ac.nz

'School of Nursing, University of Auckland, Private Bag 92019, Auckland Mail Centre, Auckland 1142, New Zealand

${ }^{3}$ Cancer Services, Princess Alexandra Hospital, 199 Ipswich Rd, Woolloongabba, QLD 4102, Australia

Full list of author information is available at the end of the article
}

comprehensive geriatric assessment (CGA) data obtained in the solid tumour chemotherapy setting.

'Frailty' is the accumulation of multiple physical and psychosocial deficits in the older person [7]. Frail older people have diminished capacity to compensate for stressors compared to people of the same chronological age; implying a state of elevated risk in the context of treatment decision-making [8]. The frailty index (FI), which is a continuous measure ranging from 0 to a theoretical maximum of 1.0 , is calculated by dividing the number of deficits identified in the CGA by the total number of variables measured. The FI therefore signifies the extent of deficit accumulation: the more deficits that are accumulated, the more likely that the individual is frail. For example, if a CGA measures 50 separate variables, an older person who records 10 variables as

(c) The Author(s). 2018 Open Access This article is distributed under the terms of the Creative Commons Attribution 4.0 International License (http://creativecommons.org/licenses/by/4.0/) which permits unrestricted use, distribution, and reproduction in any medium, provided you give appropriate credit to the original author(s) and the source, provide a link to the Creative Commons license, and indicate if changes were made. The Creative Commons Public Domain Dedication waiver (http://creativecommons.org/publicdomain/zero/1.0/) applies to the data made available in this article, unless otherwise stated. 
deficits will have a FI-CGA of 10/50 (or 0.2) [9]. The FI has a theoretical maximum of 1.0 [10].

The FI-CGA has many advantages. First, unlike many measures of frailty (such as the Fried Phenotype) [11] the FI-CGA does not privilege physiological variables. Like the CGA from which it is derived, it is multidimensional, articulating the psychosocial aspects of risk as much as the physical aspects [12]. This is critical in the chemotherapy context where the contribution to treatment outcomes of variables such as cognitive function, mood and level of social support could have as much weight as comorbidities. A further advantage of the FI-CGA is its flexibility: assuming the instruments used to measure variables are validated and reliable, the index can be constructed from different numbers and types of variables that are relevant to specific contexts. Research suggests that, provided a minimum number of deficits are chosen (about 30), variables can be selected at random and still yield comparable results on risks of adverse outcomes [13]. Third, irrespective of the composition of the CGA, its variables can contribute to a summary outcome measure, usually taken as continuous, that could flag the degree of risk associated with standard chemotherapy treatment and thereby enable chemotherapy to be tailored to the older person's identified deficits. The advantage of this is precision: the FI-CGA is far more precise than the dichotomous or interval-level risk stratifications that are common in the geriatric oncology field.

An FI was recently generated from CGA data $(n=$ 1418) in the acute hospital setting, demonstrating that every 0.1 increase in the FI-CGA was associated with a doubling of mortality (OR: 2.05 [95\% CI 1.78-2.48]) [14]. The construct validity of the FI-CGA was further confirmed in the chronic kidney care context $(n=110)$ [8], where the FI-CGA increased according to the severity of kidney disease $(p=0.04)$. In the study reported in this paper, we used CGA data to generate an FI relevant to the acute chemotherapy setting for the first time.

\section{Methods}

\section{Aim}

The overall aim of this prospective cohort study was to determine whether a clinically feasible and useful FI-CGA could be developed to predict chemotherapy decisions and survival in older patients with solid tumours.

The objectives were, in patients aged 65 years and over, to:

1. Develop an FI derived from a standardised comprehensive geriatric assessment process.

2. Compare FI-CGA assessments with:

a. Assessments of 'fit' and 'vulnerable' derived from the Vulnerable Elders Survey-13 [15]. b. Assessments of 'fit', 'vulnerable' and 'frail' derived from blinded physician judgements.

c. Documented treatment decisions (dose alterations during treatment, treatment completion), and survival over a period of 3.5 years of follow-up from date of CGA assessment.

3. Determine the clinical feasibility and utility of the CGA-derived FI in the oncology context.

Ethical approval was obtained from the Human Research Ethics Committee (HREC) - Metro South Health Service District (HREC/09/QPAH/269) prior to undertaking the study, and approval from the relevant statutory authority to obtain death registry data (where this was not recorded in the medical record). The study was undertaken according to the Strengthening the Reporting of Observational studies in Epidemiology (STROBE) guidelines [16].

\section{Setting and participants}

Princess Alexandra Hospital is a publicly-funded teaching hospital in Brisbane, Australia. It services a large geographic area of urban, rural and remote Queensland, providing chemotherapy to approximately 2000 individual cancer patients each year. Consecutively-recruited eligible patients were aged 65 years or over at the time of diagnosis; were diagnosed with a new primary or recurrence of a solid tumour; were referred for potential chemotherapy; and were able to understand conversational English. Treatments administered in addition to chemotherapy (e.g. radiotherapy and haematopoietic stem cell or bone marrow transplants) complicate treatment decision-making. Hence patients were not eligible if they had a blood cancer, were scheduled to undergo chemo-radiation, or were, in the opinion of the treating oncologist, unable to provide informed consent. Participants were recruited before treatment decisions had been made, therefore eligible participants enrolled in the study may have been prescribed a range of chemotherapy regimens (including curative and palliative regimens, first- and second-line treatments), or no treatment.

\section{Procedure}

Participants were recruited over 30 months from mid2013 to December 2015, with longitudinal data collection ceasing by December 2016. Patients were identified by their treating oncologist and referred to the project officer, who screened for eligibility and obtained informed consent. The CGA was administered by a nurse extensively trained in geriatric assessment. The ideal in geriatric oncology is a tool that can predict chemotherapy outcomes at baseline and guide physicians and patients in their subsequent treatment decisions. Hence the CGA was administered once only, before the patient 
commenced treatment. Other baseline and prospective medical data including scheduled chemotherapy, chemotherapy modifications per cycle, treatment outcomes (scheduled therapy completed/not completed) and survival for up to 3.5 years of follow-up were drawn from the electronic medical record.

To obtain the blinded oncologists' assessments of fitness for chemotherapy, the electronic chemotherapy prescribing system was configured with a pop-up questionnaire asking the oncologist to notate whether they believed, on the basis of their clinical examination, the patient was 'fit' for, 'vulnerable' to, or too 'frail' for standard adult chemotherapy. The oncologists were briefed on the precise meaning of these operational definitions, which were also built in to the pop-up questionnaire. Table 1 outlines the operational definitions used in the study.

\section{Measures}

The CGA instruments used in this study are detailed in Table 2. A Frailty Index (FI) was calculated from CGA data based on the accumulated deficits model [13] using a well-defined methodology [8]. Variables can be considered as deficits and contribute to the FI if they are associated with health status, cover a range of systems, and their prevalence generally increases with age but does not saturate (i.e., have a high prevalence so as to be almost ubiquitous) [14]. In summary, data collected as part of the CGA assessment were coded as deficits across domains of physical and cognitive functioning, nutrition, mood, basic and instrumental activities of daily living, and comorbidities (total number of deficits $=42$ ) to construct an FI based on the CGA (FI-CGA).
Participants were classified as Vulnerable/Not Vulnerable using the Vulnerable Elders Survey-13 (VES-13), a validated measure to identify older people at risk of functional decline and death [15]. The treating oncologist also assessed participants as Fit, Vulnerable or Frail based on the operational definitions outlined in Table 1. Treatment decisions comprised dose alterations of $>10 \%$ during treatment and treatment completion, with the remaining treatment outcome comprising median survival. These terms are operationally defined in Table 1.

Demographics (age, gender and socioeconomic status, living arrangements and social support such as home help) and chart data including body mass index, concomitant medications and tumour type and stage were collected. All patients were followed-up for up to 3.5 years (depending on the date of their enrolment in the study) through chart data and consultation with the State death registry after baseline assessment to determine survival.

\section{Data analysis}

Data were analysed using the Statistical Package for the Social Sciences version 23.0 [17]. Frequency distributions were used to describe the data, with proportions calculated as percent of available data. For concurrent validity, the relationship of the FI-CGA to other measures of vulnerability (VES-13 or Doctor Assessment) was tested using parametric or non-parametric tests, depending on distribution of the data, and effect sizes calculated $(\mathrm{r}=\mathrm{Z} / \mathrm{sqrt}[\mathrm{N}])$ and interpreted [18]. To investigate the predictive ability of the baseline FI on treatment decisions, regression (logistic or ordinal) was

Table 1 Operational definitions

\begin{tabular}{ll}
\hline Variable & Definition \\
\hline Fit & Should tolerate standard adult cancer therapy in addition to the anti-emetic, growth factor, superhydration \\
& and other supportive therapies usually scheduled with standard adult cancer therapy, with no modification \\
& or abandonment of the prescribed regimen.
\end{tabular}

Vulnerable

Frail

Standard adult dose (SAD)

Dose alteration

Treatment completion
Not likely to tolerate standard adult cancer therapy without requiring subsequent modification or abandonment of the prescribed regimen, but should tolerate an individually tailored anti-cancer treatment, plus supportive therapies. This might include treatment on an inpatient basis, and/or molecular-targeted therapy and/or reduction of cytotoxic drug in terms of dose, cycle or frequency at the oncologist's discretion.

All functional reserves invested in basic survival, patient may not have any additional resources to cope with the stressors induced by cancer treatments. Hence supportive, palliative, molecularly-targeted and hormone modification therapies are not precluded; however high-toxicity therapies should be excluded.

The facility where the study was conducted has well-defined chemotherapy guidelines developed by consultant oncologists with reference to the latest meta-analyses, systematic reviews, randomised controlled trials, and international and national guidelines. The SAD for each drug in each protocol is determined with reference to these resources using predefined weight-based, body surface area-based, or absolute or renal function-based dose rates. Each hospital cancer protocol is recorded in, and administered according to, the electronic chemotherapy prescribing system to ensure facility standardisation and consistent protocol delivery.

Any anticancer drug in any regimen that was altered by $10 \%$ or more during the course of treatment.

Is dichotomised to represent patients who:

1. Completed all cycles of treatment prescribed at baseline, or

2. Were not prescribed treatment due to patient decision or patient health, or withdrew prematurely from treatment due to toxicity, death, or patient decision. 
Table $\mathbf{2}$ Creation of Frailty Index from assessment tools

\begin{tabular}{|c|c|c|c|}
\hline Assessment tools & Rigour in older cancer patients & Variables used to code deficits for $\mathrm{FI}$ & Deficit Total \\
\hline $\begin{array}{l}\text { Cumulative Index Scale- } \\
\text { Geriatrics (CIRS-G) }\end{array}$ & $\begin{array}{l}\text { Confirmed criterion validity [27], good } \\
\text { concurrent construct and validity [27], } \\
\text { intrarater reliability } r=0.83 \text { and interrater } \\
\text { reliability } r=0.81[27,28]\end{array}$ & $\begin{array}{l}\text { - Comorbidities coded as deficit }(=1) \text { for each } \\
\text { comorbidity up to a maximum of } 12\end{array}$ & 12 \\
\hline $\begin{array}{l}\text { Malnutrition Screening } \\
\text { Tool (MST) }\end{array}$ & $\begin{array}{l}\text { Sensitivity 100\% [29]; specificity 81-92\% [29]; } \\
\text { PPV 0.4-0.8[29]; NPV = 1.0[29]; IRR good [29] }\end{array}$ & $\begin{array}{l}\text { - BMI Weight }(\mathrm{kg}) / \text { Height }\left(\mathrm{m}^{2}\right) \text { outside normal } \\
\text { range }(<22 \text { or }>27)=1 \\
\text { - Weight loss } \geq 6 \mathrm{~kg}=1 \\
\text { - Decreased appetite }=1\end{array}$ & 3 \\
\hline $\begin{array}{l}\text { Standardised Mini-mental } \\
\text { State (SMMSE) }\end{array}$ & $\begin{array}{l}\text { Reliability } a=0.65-0.732[30] \text {; sensitivity } 91 \% \\
{[31] \text {; specificity } 91 \%[31]}\end{array}$ & $\begin{array}{l}\text { - Cognitive impairment } \\
\text { - Mild (MMSE 20-25) =0.5 } \\
\text { - Moderate/severe }(\text { MMSE }<20)=1\end{array}$ & 1 \\
\hline $\begin{array}{l}\text { Geriatric Depression Scale } \\
\text { (GDS) }\end{array}$ & Sensitivity 96\% [31] and specificity 88\% [31] & - GDS score $\geq 5=1$ & 1 \\
\hline \multirow[t]{2}{*}{ Modified Barthel Index (MBI) } & \multirow[t]{2}{*}{$\begin{array}{l}\text { Criterion and construct validity established } \\
\text { [32]; test-retest reliability } r=0.7[32] \text {; inter-rater } \\
\text { reliability } r=0.99[32]\end{array}$} & $\begin{array}{l}\text { - ADL (personal hygiene, bathing, feeding, } \\
\text { toileting, stairs, dressing, ambulation, } \\
\text { transfers) } \\
\text { - Minimal help required }=0.5 \\
\text { - Moderate help required /Unable to do }=1\end{array}$ & 8 \\
\hline & & $\begin{array}{l}\text { - Continence (bowel and bladder) } \\
\text { - Minimal help required }=0.5 \\
\text { - Moderate help required /Unable to do }=1\end{array}$ & 2 \\
\hline Lawton IADL Scale (IADL) & $\begin{array}{l}\text { Inter-rater reliability } r=0.85[33] \text {; reliability } \\
a=0.86[33]\end{array}$ & $\begin{array}{l}\text { - IADL (phone use, shopping, food } \\
\text { preparation, house- keeping, laundry, } \\
\text { transport, medications, finances) } \\
\text { - Minimal help required }=0.5 \\
\text { - Moderate help required /Unable to do }=1\end{array}$ & 8 \\
\hline \multirow[t]{2}{*}{ Vulnerable Elders Survey-13 } & \multirow{2}{*}{$\begin{array}{l}\text { Validity established }[15] ; \text { reliability a }=0.90[15] \\
\text { sensitivity } 90 \% \text { and specificity } 70 \%[15]\end{array}$} & - Self-rated health fair/poor $=1$ & 1 \\
\hline & & $\begin{array}{l}\text { - Physical performance measures (stooping, } \\
\text { lifting, reaching, writing, walking, housework) } \\
\text { - A little/some difficulty } \\
\text { - A lot of difficulty/Unable to do }\end{array}$ & 6 \\
\hline
\end{tabular}

performed, adjusting for age and gender, to calculate an odds ratio (OR) with 95\% confidence interval (CI). The FI was categorised into groups at 0.1 intervals for ease of interpretation of the OR as the likelihood of the outcome for each 0.1 increase in the FI [19]. Kaplan Meier survival analysis assessed cumulative survival by frailty group, dichotomised as 'fit' or 'frail' and based on an established FI-CGA cut-point of $\leq 0.25$ [13].

\section{Results}

One hundred and seventy five consecutively recruited patients consented to the CGA process. Participants were predominantly male $(n=108 / 61.7 \%)$, with females making up roughly one third of the cohort $(n=67 /$ $38.3 \%)$. The mean age was 72.0 years $(S D=5.2$, Range $=$ 65-86). The majority of participants were married or living in a de facto relationship ( $n=122 / 69.7 \%)$, with the remainder not partnered. Almost all participants were pensioners ( $n=157 / 89.7 \%)$, though a small number were employed $(n=9 / 5.1 \%)$ or self-funded retirees $(n=8$ / $4.6 \%)$. Lung tumours $(n=51,29.1 \%)$ and colorectal tumours $(n=42,24.0 \%)$ were the most frequent cancer diagnoses recorded. The mean time to death was 9.15 months $(\mathrm{SD}=7.712$ ), range 0 to 33 months.
The FI-CGA could be calculated on all patients by adjusting the denominator to account for missing data. In this study $154(88 \%)$ had complete data (denominator $=$ 42), 19 (11\%) had 1 missing deficit component (denominator $=41$ ) and 2 patients had denominators of 40 and 39, to account for missing data. The index had a right-skewed distribution, with mean (SD) of $0.31(0.14)$ and median (IQR) of $0.27(0.21-0.39)$. Only $1 \%$ of the sample had an FI-CGA more than 0.75. Similar limits to deficit accumulation have been reported in community-dwelling and inpatient populations, suggesting that there is a demonstrable ceiling to the number of health problems that people can tolerate [20]. While the mean FI increased by age group (6569: $0.29 ; 70-74: 0.31 ; 75-79: 0.32 ; \geq 80: 0.33)$ these differences were not significant, nor were there significant differences based on sex. Characteristics of the patients are shown in Table 3.

As shown in Table 4, descriptive statistics showed that the Vulnerable group as measured by the VES-13 had higher median (IQR) FI (0.42 [0.36-0.52]) than the Not Vulnerable group (0.24 [0.19-0.29], Mann-Whitney U = $679.5, \mathrm{Z}=-8.86, p<0.001, r=-0.67)$. For the clinician assessment, a Kruskal-Wallis test was found to be statistically significant $\left(\mathrm{X}^{2}(2)=27.4, \mathrm{p}<0.001\right)$. Post hoc-tests 
Table 3 Characteristics of the study sample

\begin{tabular}{|c|c|}
\hline Characteristic & Total $N=175$ \\
\hline Age mean (SD) & $72.0(5.2)$ \\
\hline \multicolumn{2}{|l|}{ Age groups } \\
\hline$-65-69$ & $72(41.1)$ \\
\hline$-70-74$ & $46(26.3)$ \\
\hline$-75-79$ & $41(23.4)$ \\
\hline$-\geq 80$ & $16(9.1)$ \\
\hline \multicolumn{2}{|l|}{ Sex } \\
\hline - Males & $108(61.7)$ \\
\hline - Females & $67(38.3)$ \\
\hline Comorbidities median (IQR) & $7(5-7)$ \\
\hline BMI median (IQR) & $26.2(23.1-30.6)$ \\
\hline \multicolumn{2}{|l|}{ Malnutrition (MST) range $0-5$} \\
\hline - Low risk (0-1) & $82(46.9)$ \\
\hline - Medium risk (2-3) & $69(39.4)$ \\
\hline - High risk (4-5) & $24(13.7)$ \\
\hline \multicolumn{2}{|l|}{ Cognition (SMMSE) range 0-30 } \\
\hline - Normal (26-30) & $156(89.7)$ \\
\hline - Mild impairment (20-25) & $15(8.6)$ \\
\hline - Moderate impairment (14-19) & $2(1.1)$ \\
\hline - Severe impairment $(<14)$ & $1(0.6)$ \\
\hline \multicolumn{2}{|l|}{ Depression (GDS) range 0-15 } \\
\hline - Little/no risk $(<5)$ & $122(70.9)$ \\
\hline - Probable risk ( $\geq 5$ ) & $50(29.1)$ \\
\hline Modified Barthel Index median (IQR) & $98(93-100)$ \\
\hline Frailty Index median (IQR) & $27(0.21-0.38)$ \\
\hline Fit $(\mathrm{Fl} \leq 0.25)$ & $81(46.3)$ \\
\hline Frail $(\mathrm{FI}>0.25)$ & $94(53.7)$ \\
\hline
\end{tabular}

Figures represent number (\%) unless otherwise specified

(with Bonferroni correction) showed that the median (IQR) effect size for FI between Fit (0.24 [0.19-0.32]) and Vulnerable $(0.30[0.22-0.43])$ was small $(r=0.29)$ although significant $(p=0.001)$ and between Fit $([0.24$ $(0.19-0.32])$ and (Frail 0.38 [0.29-0.61]) was medium $(r=0.43)$ and also significant $p<0.001$.

In ordinal regression, baseline frailty as determined by the FI-CGA was also associated with treatment plan (OR 1.65; 95\% CI 1.32-2.05). Those who completed the treatment plan (highest level) were the least frail (FI median (IRQ): 0.24 [0.15-0.35]), while those whose treatment plan was terminated (middle level) (FI median (IRQ): 0.27 [0.21-0.36]), or not planned (lowest level) (FI median (IRQ): $0.40(0.30-0.56))$ were progressively frailer. There was no significant relationship between change in standard adult dose during treatment and FI-CGA (Table 4).
Of the 174 patients with follow-up data, 108 died during the follow-up period, 77 of whom died in the first year post-assessment. Figure 1 indicates a trend for better cumulative survival in the 'fit' compared with the 'frail' group using the FI-CGA cut-point of $\leq 0.25$ to categorise the 'fit' group [13] (Log Rank (Mantel-Cox) = 3.67; $p=0.055)$.

\section{Discussion}

The results demonstrate that the FI-CGA is a clinically feasible tool to predict chemotherapy outcomes in older patients with solid tumours. The No Plan group was significantly frailer than the Treatment Terminated or Treatment Completed groups (i.e., those patients at baseline who were, in medical opinion, able to tolerate chemotherapy). There was also a trend for patients classified as frail by the FI-CGA to be more likely to die in the study follow-up period. The FI-CGA also demonstrated good construct validity against fitness and vulnerability as measured by the VES-13, which has previously been shown to be highly predictive of impaired functional status in older patients with cancer [21]. There was also a significant association between subjective assessments of frailty by the treating oncologists compared with objective measurement of frailty using the FI-CGA. We acknowledge that this result in this study is an outcome of experienced oncologists working in a highly-specialised tertiary facility. Subjective assessment or 'eyeballing' patients to estimate frailty status, however, is generally fraught with difficulties and tends to be inconsistent [22].

Several good studies have tested whether elements of the CGA can rapidly predict selected chemotherapy outcomes such as toxicity [1, 3, 23]. To the best of our knowledge, this is the first study to derive an FI from the CGA to predict a range of significant clinical outcomes in older chemotherapy patients. A great deal of research in this cohort has also focused on rapid screening of frailty in order to select patients who need much more detailed assessment by way of CGA [24]. However, these simple frailty screening measures are not considered to have the discriminative ability to identify patients who might need further assessment before chemotherapy decisions are made [24, 25].

The FI-CGA demonstrated adequate discriminative ability in the study context, in that it identified patients for whom cancer treatment was also not deemed medically-appropriate. Our approach of deriving an FI from the CGA might therefore be useful in other oncology programs where treatment planning decisions are needed. It should be emphasised that the FI-CGA should not be used to discriminate against patients being assigned cancer treatment. Rather, it should be used to identify patients who might lack the reserves to cope 
Table 4 Association with frailty index

\begin{tabular}{|c|c|c|c|c|}
\hline Assessments & $N=175$ & FI median (IQR) & Effect Size & $p$ value \\
\hline \multicolumn{5}{|l|}{ VES Assessment } \\
\hline Not vulnerable & $112(64.0 \%)$ & $0.24(0.19-0.29)$ & $r=0.67$ & $<0.001$ \\
\hline Vulnerable & $63(36.0 \%)$ & $0.42(0.36-0.52)$ & & \\
\hline \multicolumn{5}{|l|}{ Dr Assessment } \\
\hline Fit & $90(53.3 \%)$ & $0.24(0.19-0.32)$ & Reference & \\
\hline Vulnerable & $52(30.8 \%)$ & $0.30(0.22-0.43)$ & $r=0.29$ & 0.001 \\
\hline Frail & $27(16.0 \%)$ & $0.38(0.29-0.61)$ & $r=0.43$ & $<0.001$ \\
\hline Treatment Decisions & $N=175$ & FI median (IQR) & OR $(95 \% \mathrm{Cl})$ & \\
\hline \multicolumn{5}{|l|}{ Treatment plan } \\
\hline Completed & 46 (26.9\%) & $0.24(0.15-0.35)$ & $1.65(1.32-2.05)$ & $<0.001$ \\
\hline Terminated & $94(55.0 \%)$ & $0.27(0.21-0.36)$ & & \\
\hline Not planned & $31(18.1 \%)$ & $0.40(0.30-0.56)$ & & \\
\hline \multicolumn{5}{|l|}{ Drug alteration } \\
\hline$<10 \%$ & $87(61.7 \%)$ & $0.27(0.21-0.35)$ & $0.98(0.71-1.28)$ & 0.89 \\
\hline$\geq 10 \%$ & $54(38.3 \%)$ & $0.26(0.20-0.38)$ & & \\
\hline
\end{tabular}

with standard adult chemotherapy and who might benefit from individually-tailored supportive therapies, or geriatrician and allied health input before treatment decisions are made. In our clinical experience, these approximate to the $36 \%$ of participants who crudely screened as 'vulnerable' by way of the VES-13; the $31 \%$ deemed 'vulnerable' by physicians before treatment commenced; and the $33 \%$ scoring between $>0.25$ to 0.4 on the FI continuum. This group is perhaps the most at-risk in terms of under- or over-treatment, and most in need of careful treatment decisions. Using the FI-CGA to flag these older patients at-risk, and exploring evidence-based treatment algorithms and referral pathways for this group, features in our research agenda.

Importantly, it is possible to derive the FI rapidly once a CGA is undertaken. For instance, it has recently been

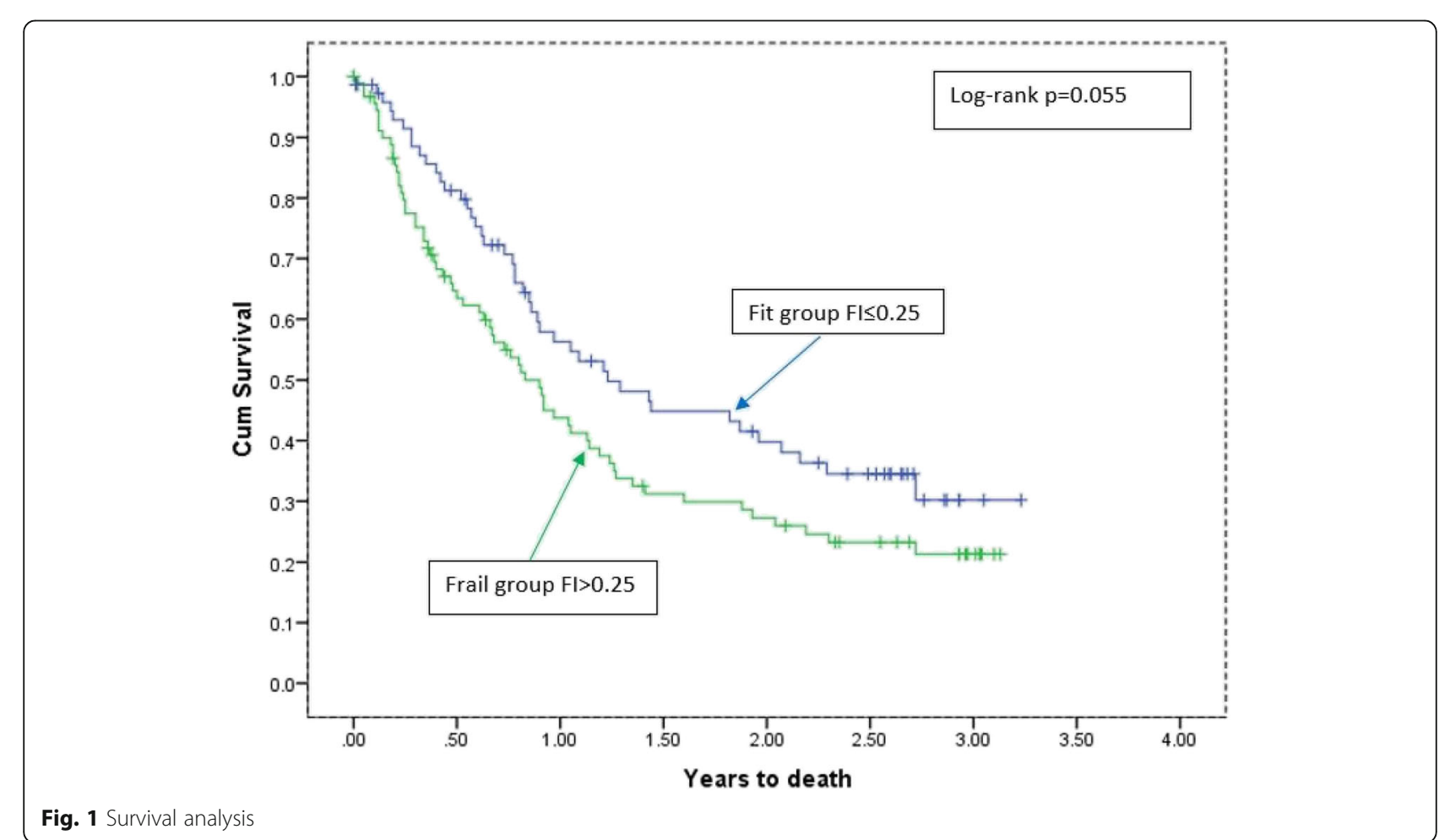


demonstrated that an FI can be derived from routinelycollected patient electronic records [26]. Accordingly, using an electronically derived FI-CGA would be particularly advantageous in the oncology setting if CGA is already standard practice.

The strength of this study is that CGAs were available for all study participants and hard clinical outcomes relating to the toleration of chemotherapy, not just mortality, were collected. Like all studies, it had limitations. Collection of data through chart review might have underestimated some outcomes. For example, cancer staging, which could have affected the outcomes, could not be accounted for in the analysis, because these data were often not documented in the medical record. However, ready access to the linked State death registry ensured that mortality outcomes were accurate when death was not documented in the patient record. While a pragmatic decision was made to include all-comers in this relatively small sample from one clinic to reflect the reality of our clinical practice, a more rigorous design would restrict the sample to one tumour type, chemotherapy-naïve patients and preferably, to one chemotherapy protocol. Due to relatively small sample size it was not possible to adjust for possible confounders, which could have biased study results and limited the generalisability of the study. Nonetheless, because of its ability to be derived from any CGA, the FI-CGA can potentially be applied across multiple oncology settings such as surgery, radiotherapy and haematology. In turn, this will facilitate comparative research.

\section{Conclusion}

Despite their over-representation in cancer populations, the older person's cancer treatment needs are not well-understood. They are often at risk of over- and under-treatment. Precise determination of risk in older people being considered for chemotherapy is critical to the development of individualised care pathways and management algorithms. Frailty is an excellent indicator of the older patient's physiological reserves. The results of this exploratory work indicate that a frailty index derived from CGA inputs can potentially contribute to accurate assessment of treatment outcomes in the chemotherapy setting.

\begin{abstract}
Abbreviations
CGA: Comprehensive geriatric assessment; Fl: Frailty index; HREC: Human Research Ethics Committee; STROBE: Strengthening the Reporting of Observational Studies in Epidemiology; VES-13: Vulnerable Elders Survey-13
\end{abstract}

\section{Funding}

Funding was supplied from a Princess Alexandra Hospital Research Foundation Grant.

\section{Availability of data and materials}

As the data are from a single centre and able to be re-identified, ethical considerations (including the conditions of ethical approval) preclude sharing the raw data beyond the research team. The data are stored in a secure repository with the Principal Investigator and will be maintained for a defined period as prescribed by ethical approval.

The datasets used and/or analysed during the current study are available from the corresponding author on reasonable request.

\section{Authors' contributions}

AM - study design, writing of paper, data analysis and interpretation. NP - study design, primary data analysis, data interpretation, contributed to all drafts of paper. KG - data collection and cleaning, contribution to manuscript. RB - data collection, paper review. EW - study design, paper review. PY - study design, paper review. $\mathrm{RH}$ - study design, data interpretation, and paper review. All authors read and approved the final manuscript.

\section{Ethics approval and consent to participate}

Ethical approval and consent for the study was given by the Metro South Health Human Research Ethics Committee, (HREC/09/QPAH/269).

We verify that patients provided written and informed consent to participate in this study.

\section{Consent for publication}

Not applicable.

Competing interests

The authors declare that they have no competing interests.

\section{Publisher's Note}

Springer Nature remains neutral with regard to jurisdictional claims in published maps and institutional affiliations.

\section{Author details}

${ }^{1}$ School of Nursing, University of Auckland, Private Bag 92019, Auckland Mail Centre, Auckland 1142, New Zealand. ${ }^{2}$ School of Nursing, Queensland University of Technology, Victoria Park Rd, Kelvin Grove, QLD 4059, Australia. ${ }^{3}$ Cancer Services, Princess Alexandra Hospital, 199 Ipswich Rd, Woolloongabba, QLD 4102, Australia. ${ }^{4}$ Centre for Research in Geriatric Medicine, University of Queensland, Level 2, Building 33, Princess Alexandra Hospital, 199 Ipswich Rd, Woolloongabba, QLD 4102, Australia.

Received: 9 January 2018 Accepted: 7 September 2018

Published online: 14 September 2018

\section{References}

1. Extermann M, Boler I, Reich RR, Lyman GH, Brown RH, DeFelice J, Levine RM, Lubiner ET, Reyes P, Schreiber FJ, et al. Predicting the risk of chemotherapy toxicity in older patients: the chemotherapy risk assessment scale for highage patients (CRASH) score. Cancer. 2012;118(13):3377-86.

2. Hurria A, Togawa K, Mohile SG, Owusu C, Klepin HD, Gross CP, Lichtman SM, Gajra A, Bhatia S, Katheria V, et al. Predicting chemotherapy toxicity in older adults with cancer: a prospective multicenter study. J Clin Oncol Off J Am Soc Clin Oncol. 2011;29(25):3457-65.

3. Massa E, Madeddu C, Astara G, Pisano M, Spiga C, Tanca FM. An attempt to correlate a "multidimensional geriatric assessment" (MGA), treatment assignment and clinical outcome in elderly cancer patients: results of a phase II open study. Critical Reviews in Oncology Haematology. 2008;66:75-83.

4. UK Department of Health: Cancer Services Coming of Age: Learning from the Improving Cancer Treatment Assessment and Support for Older People Project. In. Edited by UK Department of Health. London: UK Department of Health, Macmillan Cancer Support, Age UK, ; 2012.

5. Extermann M, Aapro M, Bernabel R, Cohen HJ, Droz JP, Steer C, al e: Main priorities for the development of geriatric oncology: a worldwide expert perspective. Journal of Geriatric Oncology 2011, 2(4):270-273.

6. International Society for Geriatric Oncology (SIOG): The SIOG 10 Priorities Initiative. In. Paris: SIOG; 2011.

7. Rockwood K, Song X, MacKnight C, Bergman H, Hogan DB, McDowell I, Mitnitski A. A global clinical measure of fitness and frailty in elderly people. Can Med Assoc J. 2005;173(5):489-95.

8. Hubbard RE, Peel NM, Samanta M, Gray LC, Fries BE, Mitnitski A, Rockwood K. Derivation of a frailty index from the interRAl acute care instrument. BMC Geriatr. 2015;15(1):27. 
9. Mitnitski AB, Mogilner AJ, Rockwood K. Accumulation of deficits as a proxy measure of aging. ScientificWorldJournal. 2001;1:323-36.

10. Searle SD, Mitnitski A, Gahbauer EA, Gill TM, Rockwood K. A standard procedure for creating a frailty index. BMC Geriatr. 2008;8.

11. Fried LP, Tangen CM, Walston J, Newman AB, Hirsch C, Gottdiener J, Seeman T, Tracy R, Kop WJ, Burke G, et al. Frailty in older adults: evidence for a phenotype. Journal of Gerontology: Series A Biology Sciences and Medical Sciences. 2001;56(3):M146-56.

12. Dent E, Hoogendijk EO. Psychosocial factors modify the association of frailty with adverse outcomes: a prospective study of hospitalised older people. BMC Geriatr. 2014;14

13. Rockwood K, Mitnitski A. Frailty in relation to the accumulation of deficits. The Journals of Gerontology Series A, Biological Sciences and Medical Aciences. 2007;62(7):722-7.

14. Hubbard RE, Peel NM, Smith M, Dawson B, Lambat Z, Bak M, Best J, Johnson DW. Feasibility and construct validity of a frailty index for patients with chronic kidney disease. Australasian Journal on Ageing 2015;34(3):E9-E12

15. Saliba D, Elliot M, Rubenstein LZ, Solomon DH, Young RT, Kamberg CJ, Roth C, MacLean CH, Shekelle PG, Sloss EM, et al. The vulnerable elders survey: a tool for identifying vulnerable older people in the community. J Am Geriatr Soc. 2001:49(12):1691-9.

16. von Elm E, D.G. A, Egger M, Pocock SJ, Gotzsche PC, Vandenbroucke JP: The strengthening the reporting of observational studies in epidemiology (STROBE) statement: guidelines for reporting observational studies. J Clin Epidemiol 2008, 61(4):344-349.

17. IBM Corp: The statistical package for the social sciences: IBM SPSS statistics for windows. In., 23.0 edn. Armonk, NY: IBM Corp; 2014.

18. Cohen J: Statistical power analysis for the behavioral sciences. Hillside, New Jersey: L. Erlbaum Associates; 1988.

19. Hubbard RE, Peel NM, Samanta M, Gray LC, Mitnitski A, Rockwood K. Frailty status at admission to hospital predicts multiple adverse outcomes. Age Ageing. 2017;46(5):801-6.

20. Rockwood K, Andrew M, Mitnitski A. A comparison of two approaches to measuring frailty in elderly people. J Gerontol Ser A Biol Med Sci. 2007;62:5.

21. Luciani A, Ascione G, Bertuzzi C, Marussi D, Codecà C, Di Maria G, Caldiera SE, Floriani I, Zonato S, Ferrari D, et al. Detecting disabilities in older patients with Cancer: comparison between comprehensive geriatric assessment and vulnerable elders Survey-13. J Clin Oncol. 2010;28(12):2046-50.

22. Hubbard RE, Story DA. Does frailty lie in the eyes of the beholder? Heart, Lung and Circulation. 2015;24(6):525-6.

23. Hurria A, Mohile S, Gajra A, Klepin H, Muss H, Chapman A, Feng T, Smith D, Sun C, De Glas N, et al. Validation of a prediction tool for chemotherapy toxicity in older adults with cancer. J Clin Oncol. 2016;34(20).

24. Hamaker ME, Jonker MM, de Rooij SE, Vos AG, Smorenburg CH, van Munster $B C$. Frailty screening methods for predicting outcome of a comprehensive geriatric assessment in elderly patients with cancer: a systematic review. Lancet Oncol. 2012:13:437-44.

25. Senior Adult Oncology Version 2.2013 [https://www.nccn.org/professionals/ physician_gls/pdf/senior.pdf].

26. Clegg A, Bates C, Young J, Ryan R, Nichols L, Ann Teale E, Mohammed MA, Parry J, Marshall T. Development and validation of an electronic frailty index using routine primary care electronic health record data. Age Ageing. 2016; 45(3):353-60.

27. de Groot V, Beckerman H, Lankhorst GJ, Bouter LM. How to measure comorbidity: a critical review of available methods. J Clin Epidemiol. 2003;56(3):221-9.

28. Salvi F, Miller MD, Grilli A, Giorgi R, Towers AL, Morichi $V$, et al. A manual of guidelines to score the modified cumulative illness rating scale and its validation in acute hospitalized elderly patients. Journal of the American Society of Geriatrics. 2008;56(10):1926-31.

29. Isenring E, Cross G, Daniels L, Kellett E, Koczwara B. Validity of the malnutrition screening tool as an effective predictor of nutritional risk in oncology outpatients receiving chemotherapy. Support Care Cancer. 2006;14: 1152-6.

30. Overcash JA, Beckstead J, Moody L, Extermann M, Cobb S. The abbreviated comprehensive geriatric assessment (aCGA) for use in the older cancer patient as a prescreen: scoring and interpretation. Crit Rev Oncol Hematol. 2006:59(3):205-10.
31. Kai H, Hoi SF, Wong E, Lo R, Woo J. Do pain and disability differ in depressed cancer patients? Palliat Med. 2000;14(1):11-7.

32. Cohen ME, Marino RJ. The tools of disability outcomes research functional status measures. Archives of Physical and Medical Rehabilitation. 2000 81(Suppl. 2):S21-9.

33. Graf $C$. The Lawton instrumental activities of daily living (IADL) scale. Annals of Long-Term Care. 2007;15(7):21-2.
Ready to submit your research? Choose BMC and benefit from:

- fast, convenient online submission

- thorough peer review by experienced researchers in your field

- rapid publication on acceptance

- support for research data, including large and complex data types

- gold Open Access which fosters wider collaboration and increased citations

- maximum visibility for your research: over $100 \mathrm{M}$ website views per year

At BMC, research is always in progress.

Learn more biomedcentral.com/submissions 